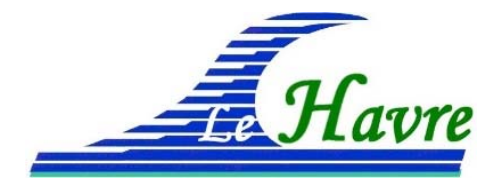

XVI ${ }^{e ̀ m e s}$ Journées Nationales Génie Côtier - Génie Civil
Le Havre, 2020

DOI:10.5150/jngcgc.2020.058 (C) Editions Paralia CFL

disponible en ligne - http://www.paralia.fr - available online

\title{
Courbes de puissance pour une hydrolienne à axe vertical de type Darrieus en milieu fini
}

\author{
Mohamed Elamine GHEDHAB ${ }^{1}$, Ikram EL ABBASSI ${ }^{1}$, \\ Rafik ABSI ${ }^{2}$, Abdel Moumen DARCHERIF ${ }^{2}$
}

\author{
1. L2MGC-Lab, ECAM-EPMI, LR2E-lab, 13 Boulevard de l'Hautil, \\ 95092 Cergy Pontoise, France. \\ m.ghedhab@ecam-epmi.com ; i.elabbassi@ecam-epmi.com \\ 2. Quartz-lab - EA 7393, ECAM-EPMI, LR2E-lab, 13 Boulevard de l'Hautil, \\ 95092 Cergy Pontoise, France. \\ r.absi@ecam-epmi.com ; m.darcherif@ecam-epmi.com
}

\section{Résumé :}

L'étude porte sur le comportement d'une hydrolienne à axe vertical de type Darrieus avec des profils de pales de type NACA 4415 en milieux infini et fini. Nous avons choisi le modèle de turbulence k- $\omega$ SST disponible dans ANSYS FLUENT (2014). D'abord le modèle a été validé en milieu infini, puis utilisé pour traiter le domaine fini en utilisant la correction bas-Reynolds (Low Reynolds Correction "LReC"). Les courbes de puissance obtenues pour différentes valeurs de la vitesse spécifique ( $\lambda$ ou TSR) ont été comparées à des résultats expérimentaux réalisées en canal (issues de la littérature). Nous avons obtenu un bon accord pour des TSR faibles $(<1.5)$, cependant pour des TSR plus important l'écart devient important avec une courbe numérique croissante contrairement à la courbe expérimentale décroissante. Nous avons effectué des simulations pour des TSR plus important (jusqu'à 4). La courbe de coefficient de puissance obtenue montre un comportement similaire à celle d'une l'éolienne du même type.

\section{Mots-clés :}

Hydroliennes, Axe vertical/Darrieus, Vitesse spécifique, Puissance, Profil de pales, Modèle de turbulence k- $\omega$ SST

\section{Introduction}

L'énergie marine, contenue dans les houles et courants, représente une source naturelle inépuisable. Les hydroliennes permettent de transformer l'énergie cinétique contenue dans l'eau en énergie mécanique (mouvement rotatif), (CARLIER et al., 2014; NGUYEN et al., 2014 ; KHAN et al., 2009). Les hydroliennes à axe vertical plus précisément de type Darrieus ont des avantages par rapport aux autres (MENCHACA ROA, 2011 ; AIT MOHAMMED, 2016). Ces dernières années, plusieurs études expérimentales et numériques ont été effectuées pour améliorer le rendement des hydroliennes et éoliennes de ce type, (BELHACHE et al., 2012). SHIONO et al., (2000) ont étudié expérimentalement l'effet de solidité ( $\sigma$ qui représente le rapport entre la 


\section{Thème 5 - Énergies et ressources marines}

surface des pales et celle du rotor, donnée par $\sigma=\mathrm{N} . \mathrm{C} / \pi . \mathrm{D}$ avec $\mathrm{N}$ est le nombre des pales, $\mathrm{C}$ la corde de la pale et $\mathrm{D}$ le diamètre du rotor). Ils ont trouvé que le couple maximal était atteint pour $\sigma=0.366$, alors que le rendement maximal de $23.5 \%$ était obtenu pour $\sigma=0.179$ et une vitesse spécifique (ou TSR, tip speed ratio) plus grande. Ils ont trouvé également que le nombre de pales a une influence sur la puissance développé. LAIN \& OSORIO (2010) ont effectué des simulations numériques 2D avec Fluent en utilisant le modèle k$\omega$ SST pour différents maillages et plusieurs pas de temps. Comme particularité, ils ont pris des "parois glissantes", c'est-à-dire que seule la composante normale de la vitesse est nulle, pour le haut et le bas du domaine avec la même vitesse d'écoulement. Les résultats obtenus à travers leurs modélisations sont très proches des résultats expérimentaux publiés dans la littérature. MENCHACA ROA (2011) et HAMMOUDI et al. (2016) ont validé leurs simulations avec les données issues des références SHIONO et al., (2000) et LAIN \& OSORIO (2010), pour une hydrolienne dans un domaine infini en utilisant des formes de pales différentes pour étudier l'influence de l'épaisseur entre NACA0015 et NACA0018, et l'effet de la cambrure pour NACA0018 et 4415. MENCHACA ROA (2011) a travaillé sur une hydrolienne de type Darrieus avec deux types d'installations libres et avec un carénage numériquement et expérimentalement. Ils ont étudié également l'influence de la valeur de la taille de la première maille au voisinage immédiat de la paroi des pales $\mathrm{y}^{+}$sur les résultats de simulation, avec 4 différents maillages et ont trouvé que pour un maillage avec $\mathrm{y}^{+} \leq 2$ les simulations donnent de bons résultats. PATEL et al., (2017) ont réalisé des expériences en canal pour étudier les performances hydrodynamiques d'une hydrolienne de type Darrieus. Ils ont utilisé 3 types de profils NACA symétriques $(0015,0018)$ et asymétrique (4415), pour différentes valeurs de solidité.

Notre étude porte sur un modèle 2D d'une hydroliennes à axe vertical de type Darrieus avec profil asymétrique NACA4415 et une solidité de 0.258. Afin de calculer les coefficients de puissance pour différentes vitesses spécifiques (ou TSR), nous utiliserons le modèle de turbulence $\mathrm{k}-\omega$ SST avec une correction bas-Reynolds (LReC) disponible dans un code CFD commercialisé (ANSYS FLUENT, 2014). Nous étudierons l'effet de la taille de la première maille au voisinage immédiat de la paroi des pales. Nous comparerons nos résultats de simulations avec les courbes de puissances théoriques et expérimentales obtenues en canal par PATEL et al., (2017).

\section{Travail réalisé}

\subsection{Réalisation de la géométrie}

La géométrie bidimensionnelle 2D (voir figure 1) est effectuée à l'aide de l'outil "Design Modeler" de ANSYS FLUENT (2014), elle est réalisée en deux domaines différents, un fixe (milieu de l'écoulement) et l'autre mobile (zone rotative de l'hydrolienne) pour pouvoir utiliser la technique de "Sliding Mesh" dans Fluent. Le profil de pale utilisé est 


\section{XVİ̀mes Journées Nationales Génie Côtier - Génie Civil \\ Le Havre 2020}

le NACA4415 (http://airfoiltools.com/airfoil/naca4digit) correspondant à un des dispositifs utilisés par PATEL et al., (2017).
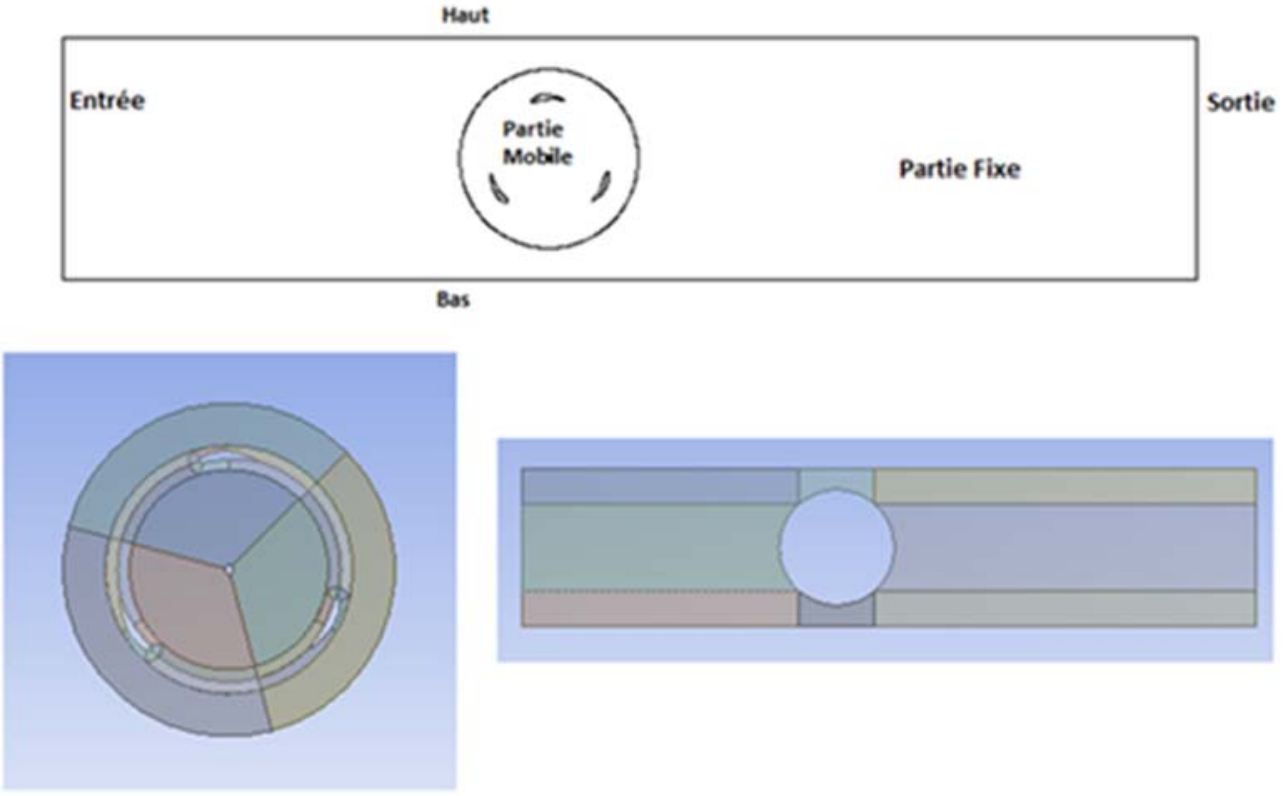

Figure 1. Géométrie du domaine étudié en 2D en vue de dessus. Cas du canal.

\subsection{Maillage}

Le maillage a été réalisé à l'aide du logiciel "Meshing" sous "Workbench". Lors de la création de la géométrie, nous avons créé des zones pour faire un maillage multi-blocs pour mieux contrôler le maillage sur tout le domaine (voir figure 2).
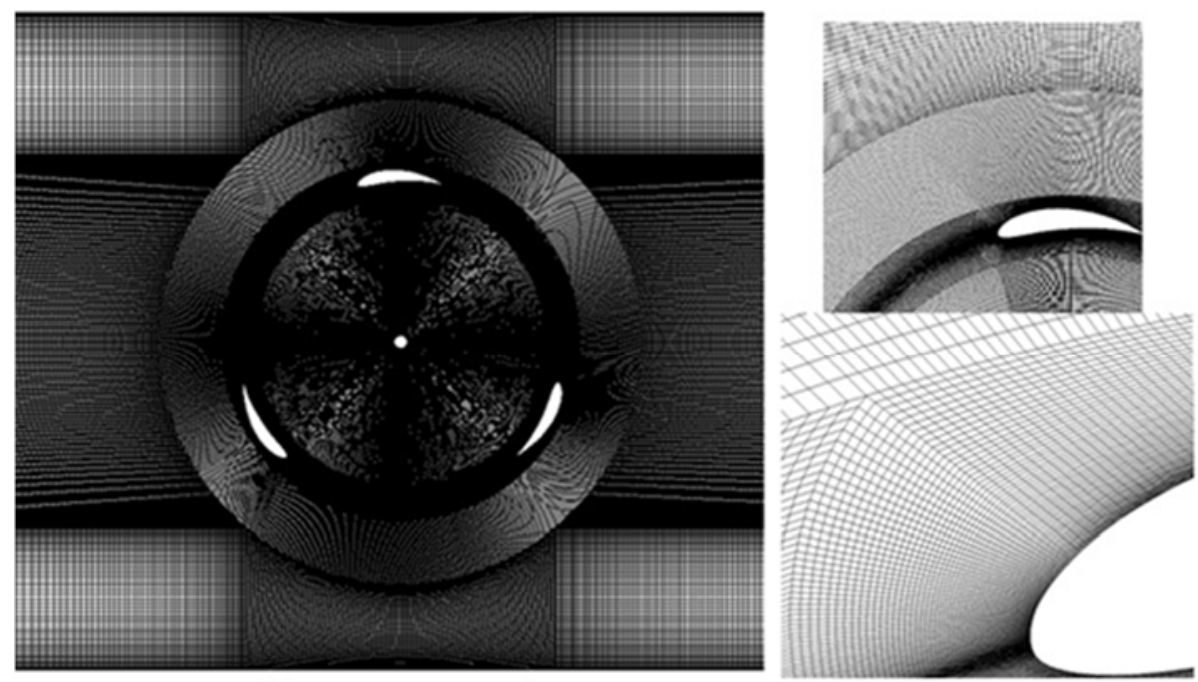

Figure 1. Maillage multizones. 


\section{Thème 5 - Énergies et ressources marines}

Afin d'avoir un bon compromis entre la taille du maillage et le coût de calcul, une étude sur l'influence du maillage a été réalisé pour la deuxième partie de cette étude.

\subsection{Simulations avec ANSYS FLUENT (2014)}

Après avoir importé le maillage créé avec "Meshing" dans ANSYS FLUENT 16.0 (2014), on choisit les paramètres adéquats avant de lancer les simulations. Pour faire tourner la partie mobile du domaine modélisé, il faut créer une interface dans "Mesh interfaces" entre les deux parties fixe et rotative. Cette étape permet l'introduction d'une vitesse de rotation pour la partie mobile. Les calculs se font en mode instationnaire, le modèle de turbulence appliqué est le modèle k- $\omega$ SST (MENTER, 1994 ; MENCHACA ROA, 2011). Le couplage vitesse-pression est assuré par l'algorithme SIMPLE (acronyme de Semi-Implicit Method for Pressure Linked Equations), les schémas de discrétisation sont au deuxième ordre, et pour bien assurer la convergence des calculs, les résidus sont fixés à $10^{-5}$ et les sous itérations temporelles sont fixées à 100 , le pas temporel choisi correspond à $1^{\circ}$ de rotation pour la turbine.

Tableau 1. Dimensions de l'hydrolienne dans le milieu infini.

\begin{tabular}{|l|l|l|l|l|l|l|l|l|}
\hline $\begin{array}{l}\text { Solidité } \\
(-)\end{array}$ & $\begin{array}{l}\text { Profil } \\
(-)\end{array}$ & $\begin{array}{l}\text { Diamètre } \\
(\boldsymbol{m})\end{array}$ & $\begin{array}{l}\text { Corde } \\
(\boldsymbol{m})\end{array}$ & $\begin{array}{l}\text { Hauteur } \\
(\boldsymbol{m})\end{array}$ & $\begin{array}{l}\text { Nombre de pales } \boldsymbol{(} \\
(-)\end{array}$ & $\begin{array}{l}\text { Vitesse } \boldsymbol{V} \\
(\mathbf{m} / \mathbf{s})\end{array}$ & $\begin{array}{l}W_{w} \\
(\mathbf{m})\end{array}$ & $\begin{array}{l}A_{s} \\
\left(\mathbf{m}^{2}\right)\end{array}$ \\
\hline 0.179 & NACA4415 & 0.175 & 0.0328 & 0.175 & 3 & 2.3 & 1.75 & 0.0306 \\
\hline
\end{tabular}

Tableau 2. Dimension de l'hydrolienne dans le milieu fini.

\begin{tabular}{|l|l|l|l|l|l|l|l|l|l|}
\hline $\begin{array}{l}\text { Solidité } \\
(-)\end{array}$ & $\begin{array}{l}\text { Profil } \\
(-)\end{array}$ & $\begin{array}{l}\text { Diamètre } \\
(\mathbf{m})\end{array}$ & $\begin{array}{l}\text { Corde } \\
(\mathbf{m})\end{array}$ & $\begin{array}{l}\text { Hauteur } \\
(\mathbf{m})\end{array}$ & $\begin{array}{l}\text { Nombre de pales } \boldsymbol{N} \\
(-)\end{array}$ & $\begin{array}{l}\text { Vitesse } \boldsymbol{V} \\
(\mathbf{m} / \mathbf{s})\end{array}$ & $\begin{array}{l}W_{w} \\
(\mathbf{m})\end{array}$ & $\begin{array}{l}\boldsymbol{H}_{w} \\
(\mathbf{m})\end{array}$ & $\begin{array}{l}A_{s} \\
\left(\mathbf{m}^{2}\right)\end{array}$ \\
\hline 0.258 & NACA4415 & 0.37 & 0.1 & 0.15 & 3 & 0.463 & 0.75 & 0.3 & 0.0555 \\
\hline
\end{tabular}

Pour les conditions aux limites, les pales sont considérées comme parois (walls), le haut et le bas (i.e. le bas et haut correspondent respectivement à droite et à gauche dans la réalité), du domaine d'écoulement (partie fixe) sont considérés comme symétrie pour le milieu infini et parois pour le milieu fini. Nous avons utilisé la condition "symmetry" d'ANSYS Fluent pour que l'hydrolienne soit en milieu infini. Dans la première partie, nous avons repris les simulations réalisées par HAMMOUDI et al., (2016). Nous avons fait des simulations pour $\mathrm{TSR}=1$ et $\mathrm{TSR}=2$, les dimensions prises sont identiques à celles de MENCHACA ROA (2011), (voir tableau 1). La largeur $\mathrm{W}_{\mathrm{w}}$ du domaine est égale à 10 fois le diamètre de l'hydrolienne. Pour la deuxième partie où le milieu est fini, la géométrie est réalisée avec d'autres dimensions en $2 \mathrm{D}$, elles sont identiques à celles de l'expérience réalisé par PATEL et al. (2017), (voir tableau 2), nous avons choisi la solidité 0.258 en utilisant le même profil NACA4415 pour valider les premières simulations. 


\section{XVİ̀mes Journées Nationales Génie Côtier - Génie Civil \\ Le Havre 2020}

\section{Résultats et discussions}

\subsection{Milieu infini}

ANSYS FLUENT (2014) nous donne des coefficients de moment instantanés. La figure 3 montre les coefficients de moment instantanés pour chaque pale ainsi que le coefficient total instantané de l'hydrolienne. Nous confirmons que l'ordre des pics de coefficient de moment de chaque pale représenté par MENCHACA ROA (2011) était inversé pour les pales 2 et 3 (HAMMOUDI et al., 2016). Nous validons également par comparaison avec le résultat de LAIN ET OSORIO (2010), (figure 4). La moyenne du $\mathrm{C}_{\mathrm{M}}$ est calculée sur un tour complet de l'hydrolienne sur la zone où les oscillations deviennent régulières comme on peut le constater sur la figure 5. Le coefficient de puissance $C_{P}$ s'obtient à partir le produit du coefficient de moment et du TSR : $\mathrm{C}_{\mathrm{P}}=\lambda \mathrm{C}_{\mathrm{M}}\left(\mathrm{C}_{\mathrm{M}}=\mathrm{Mz} /(\mathrm{q}\right.$ As $\mathrm{R})=2 \mathrm{Mz} /(\rho$ $\left.\mathrm{V}_{\infty}{ }^{2} \mathrm{As}_{\mathrm{S}} \mathrm{R}\right)$ ).

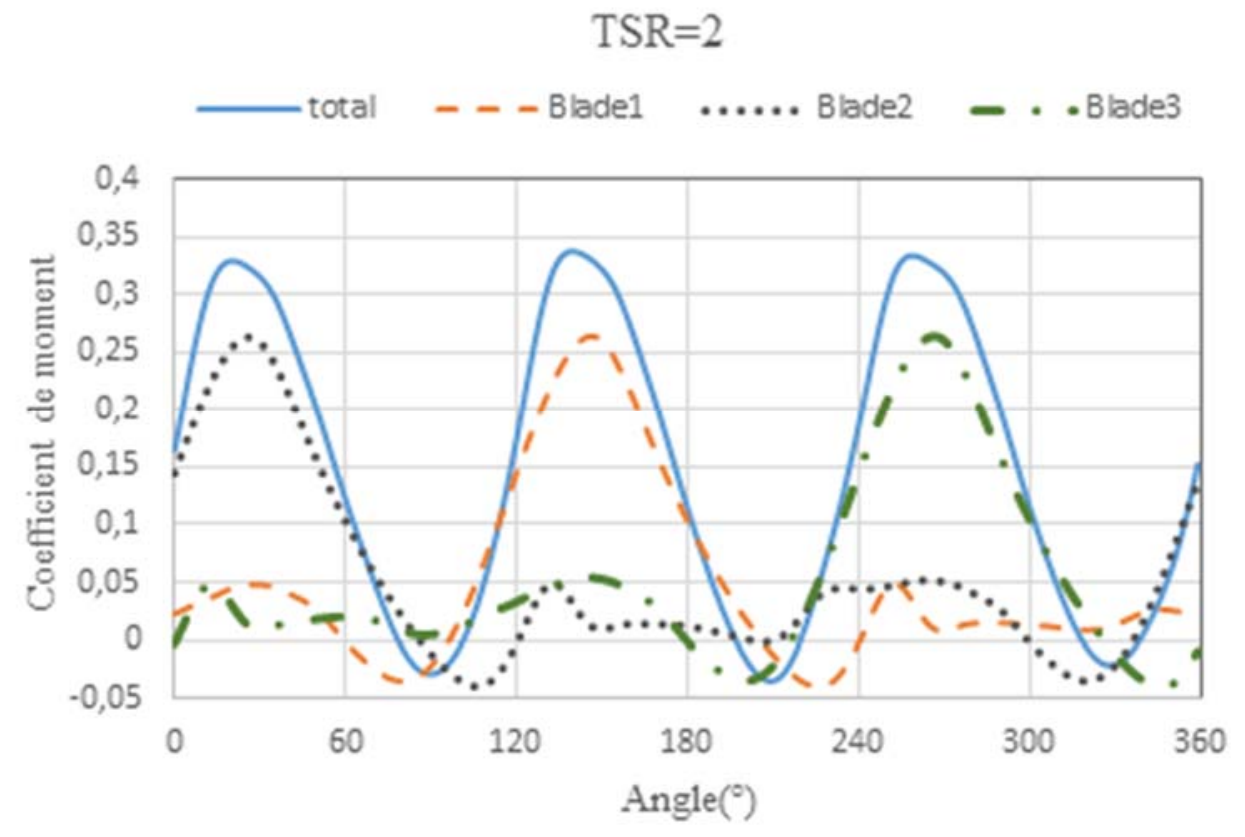

Figure 2. Coefficient de moment instantané sur un tour d'hydrolienne (un milieu infini). 


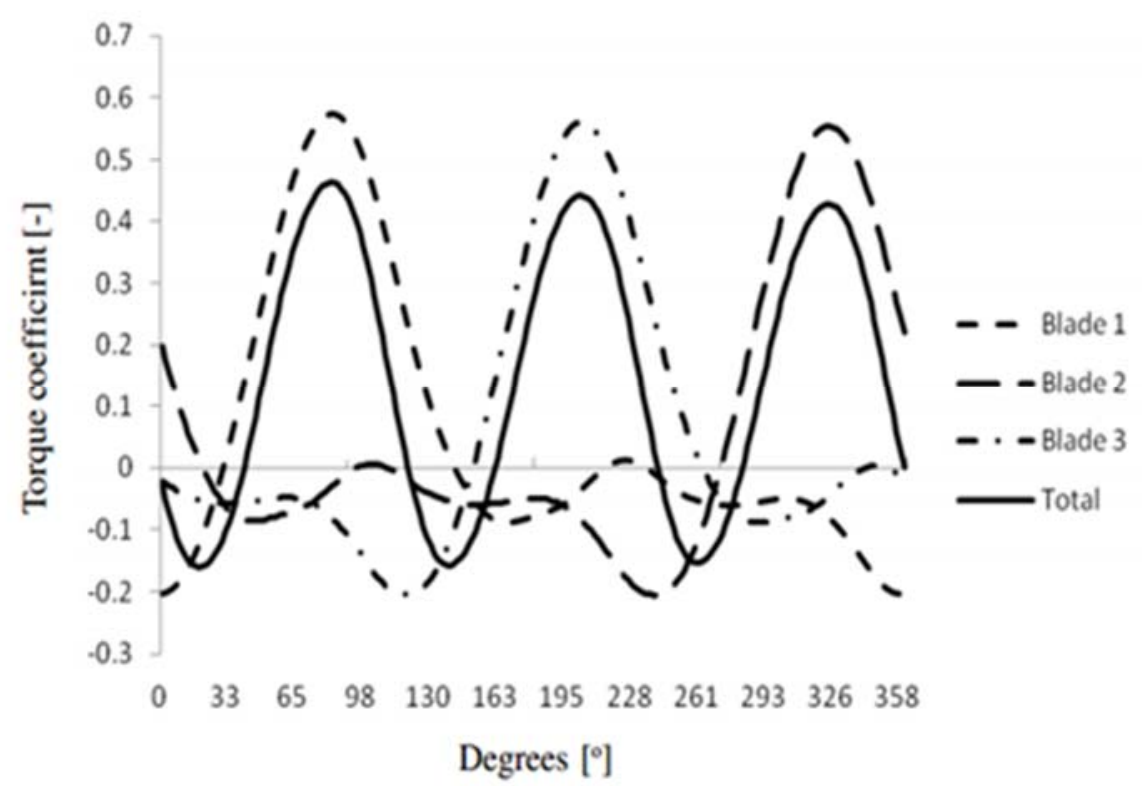

Figure 3. Coefficient de moment instantané pour chaque pale (LAIN \& OSORIO, 2010).

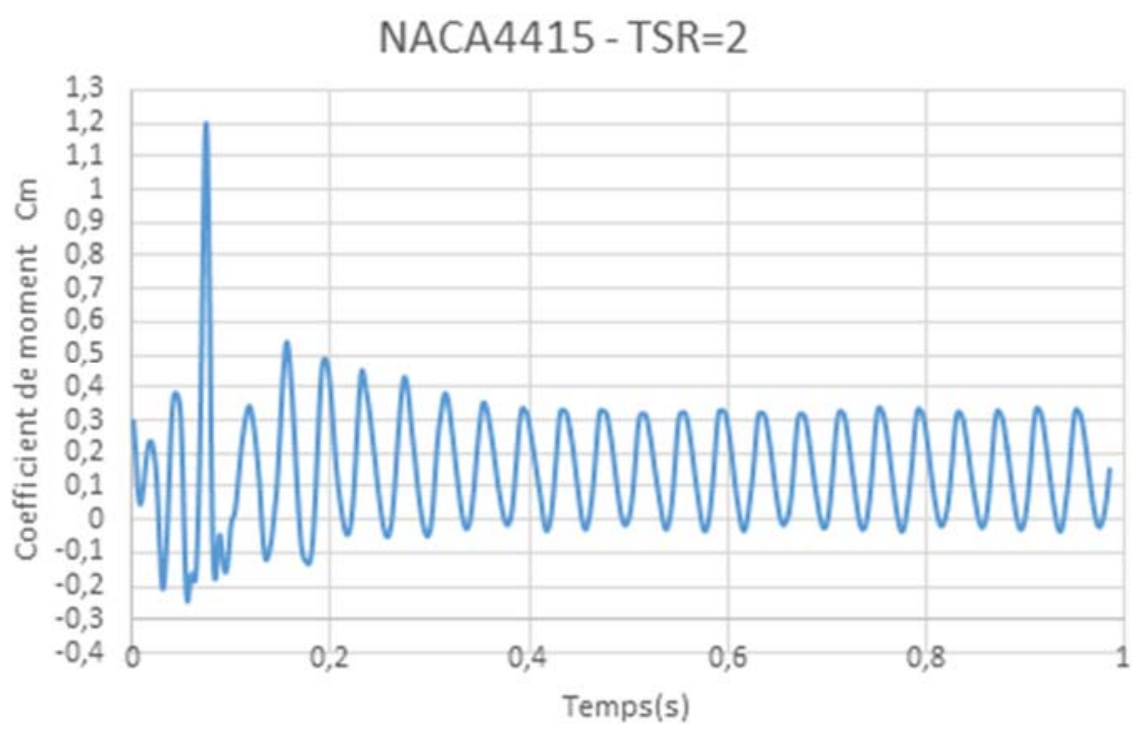

Figure 4. Coefficient de moment total instantané pour un milieu infini à TSR=2.

\subsection{Milieu fini}

Au niveau d'ANSYS FLUENT (2014), le choix du modèle k- $\omega$ SST, s'accompagne par l'activation ou pas de la correction bas-Reynolds. Cette correction nécessite un maillage raffiné avec une taille de la première maille au voisinage immédiat de la paroi des pales $\mathrm{y}+$ de l'ordre de 1 . Dans ce cas, les simulations sont donc réalisées sans loi de la paroi, la sous couche visqueuse et la zone tampon de la couche limite sont résolus. 


\section{XVIèmes Journées Nationales Génie Côtier - Génie Civil \\ Le Havre 2020}

Pour rappel, l'utilisation d'une fonction de paroi (haut-Reynolds) est possible pour les applications qui concernent l'écoulement loin des parois (tels que mélange, ...). Cependant, dans le cadre des machines tournantes (turbomachines en général et hydroliennes en particulier) où un calcul précis des forces (portances et trainées dues aux pressions dynamiques au niveau des parois) est très important, la résolution de la sous couche visqueuse est nécessaire (d'où un modèle bas-Reynolds). Pour rappel, dans la correction bas-Reynolds, l'amortissement de la viscosité turbulente $v_{t}$ est obtenue par le coefficient $\alpha^{*}: v_{t}=\alpha^{*} k / \omega$. Alors que dans les modèle "haut-Reynolds" $\alpha^{*}=1$.

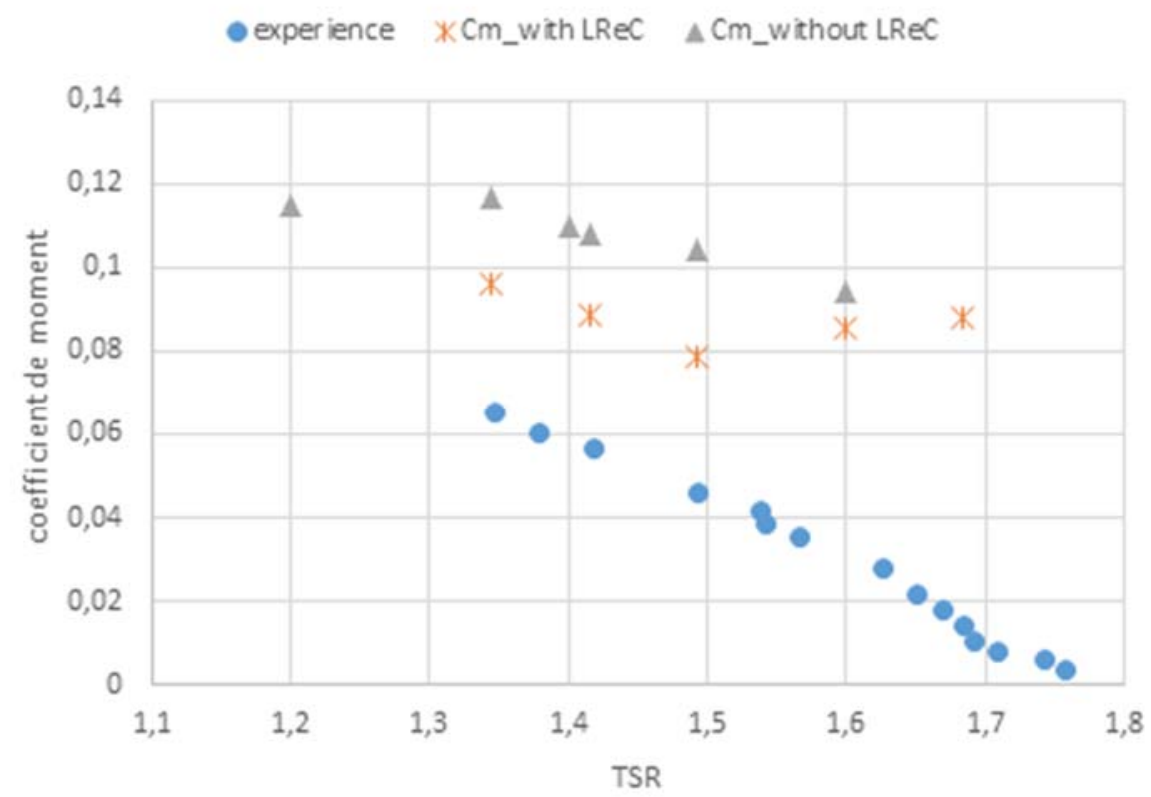

Figure 5. Comparaison entre coefficients de moment numériques avec et sans LReC (LReC : Low Reynolds Correction) et expérimentaux.

\subsubsection{Simulations sans correction bas-Reynolds (LReC)}

Nous avons commencé par réaliser des simulations avec le modèle k- $\omega$ SST en désactivant la correction bas-Reynolds ( $\mathrm{LReC}$ ). Les coefficients de moment $\mathrm{C}_{\mathrm{M}}$ ont été calculés pour différents TSR. Les résultats de simulations (figure 6, triangles) montrent une allure similaire aux résultats expérimentaux de PATEL et al. (2017) (cercles pleins). Cependant on constate un écart entre les valeurs de $\mathrm{C}_{\mathrm{M}}$ numériques et expérimentales qui est relativement constant (de l'ordre de 0.06). Compte tenu de l'écart observé, nous avons cherché son origine afin d'améliorer le résultat. Nous avons procédé dans un premier temps à une diminution de la taille de la première maille $\mathrm{y}^{+}$(accompagné d'un nombre de mailles $\mathrm{M}$ plus grand). Nous avons testé différentes valeurs du couple $\mathrm{y}^{+} / \mathrm{M}$ données par : $10 / 285150,7 / 539000$ et 2.5/484750 pour un $\mathrm{TSR}=1,416$. Les résultats pour $\mathrm{C}_{\mathrm{M}}$ montrent un écart de plus en plus important $\left(\mathrm{C}_{\mathrm{M}}\right.$ respectivement de 0,$\left.108 ; 0,12 ; 0,127\right)$ avec la 


\section{Thème 5 - Énergies et ressources marines}

valeur expérimentale $\left(\mathrm{C}_{\mathrm{M}}\right.$ exp=0.057). Ces résultats montrent que les simulations, effectuées sans activation de la fonctionnalité bas-Reynolds (sans LReC), sont réalisées au niveau d'ANSYS FLUENT (2014) avec une loi de la paroi. Dans ce cas, sans correction bas-Reynolds, la sous couche visqueuse et la zone tampon de la couche limite sont donc modélisées et pas résolus.

\subsubsection{Simulations avec correction bas-Reynolds (LReC)}

Afin d'améliorer le résultat obtenu précédemment sans correction bas-Reynolds, nous avons décidé de tester le modèle k- $\omega$ SST avec activation de LReC (ANSYS FLUENT, 2014) qui nécessite un maillage très raffiné à proximité des parois des pales. Le choix de la valeur de $\mathrm{y}^{+} \mathrm{s}$ 'est posé pour ce type de traitement. Compte-tenu des recommandations d'ANSYS FLUENT (2014) de prendre un $\mathrm{y}^{+}$de l'ordre de 1, nous avons choisi de prendre $\mathrm{y}^{+}=0,74$ ce qui donne un nombre de mailles $(\mathrm{M}=458600)$. La correction avec un modèle bas-Reynolds (Low Reynolds Correction) ainsi qu'un choix d'une valeur de $\mathrm{y}+$ faible ont permis d'avoir une amélioration du résultat. Pour un $\mathrm{TSR}=1.416$, nous obtenons $\mathrm{C}_{\mathrm{M}}=0,0883$, l'écart avec la valeur expérimentale de PATEL et al., (2017) $\left(\mathrm{C}_{\mathrm{M}}\right.$ expérimental $\left.=0.057\right)$, est réduit.

Les résultats obtenus (figure 6, étoiles) montrent que les simulations arrivent à bien reproduire qualitativement l'allure de la courbe du $\mathrm{CM}_{\mathrm{M}}$ pour des $\mathrm{TSR}<1.5$. Au-delà de cette valeur les $\mathrm{C}_{\mathrm{M}}$ numériques augmentent contrairement à celles obtenues expérimentalement (PATEL et al., 2017) où les CM continuent de décroitre jusqu'à une valeur presque nulle. D'après PATEL et al., (2017), il pourrait y avoir une perte d'information au niveau du système expérimental de mesure du $\mathrm{C}_{\mathrm{M}}$ pour des vitesses spécifiques plus importantes. En effet, les valeurs de coefficients de moment et de puissance ont été mesurées avec un système comprenant un dynamomètre et à l'aide de différentes charges sur l'arbre de la turbine. La question qu'on s'est posé concerne les valeurs expérimentales de puissance et de moment ne sachant pas si c'étaient des valeurs maximales ou moyennes. Nous n'avons pas pu avoir confirmation concernant ces valeurs expérimentales. Nous avons étudié la variation du CP pour notre hydrolienne pour des valeurs de TSR plus importante (allant jusqu'à 4). Les résultats obtenus (figure 7) montrent un comportement similaire à celle d'une l'éolienne (figure 8). La figure 8 montre un schéma type représentant la variation du coefficient de Puissance CP d'une éolienne de type Darrieus pour des TSR allant jusqu'à environ 10 (PARASCHIVOIU, 2002). Elle montre trois parties : une région de décrochage dynamique, une région de transition et une région des effets secondaires. Le Cp max (figure 7) est aux alentours de 0.4 comme c'est le cas pour l'éolienne de type Darrieus. Par contre, le CPmax est atteint plus tôt à $\mathrm{TSR}=3$. Cette courbe (figure 7) permet d'identifier les vitesses (ou conditions) de fonctionnement optimal de la turbine et connaitre le rendement maximal (meilleur rendement de l'hydrolienne environ $45 \%$ pour $\mathrm{TSR}=3$ ). 


\section{XVIèmes Journées Nationales Génie Côtier - Génie Civil \\ Le Havre 2020}

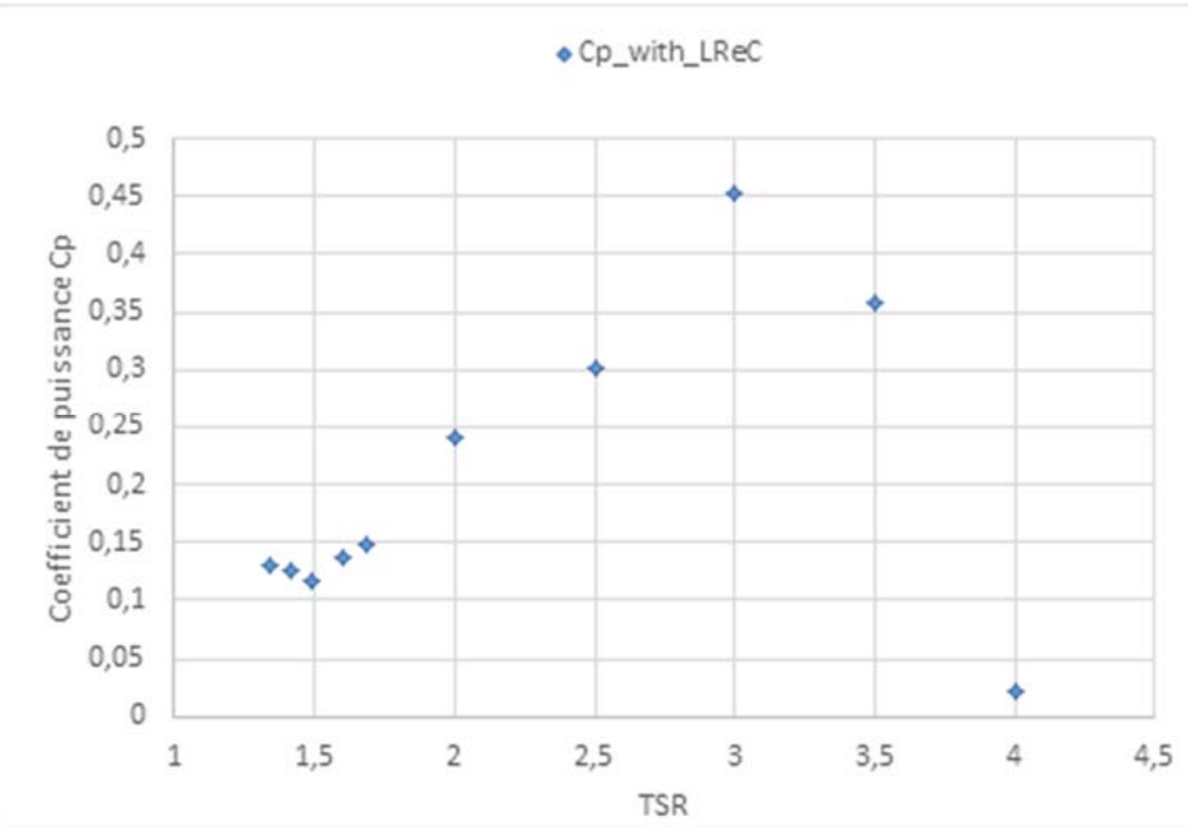

Figure 6. Coefficient de puissance pour un milieu fini.

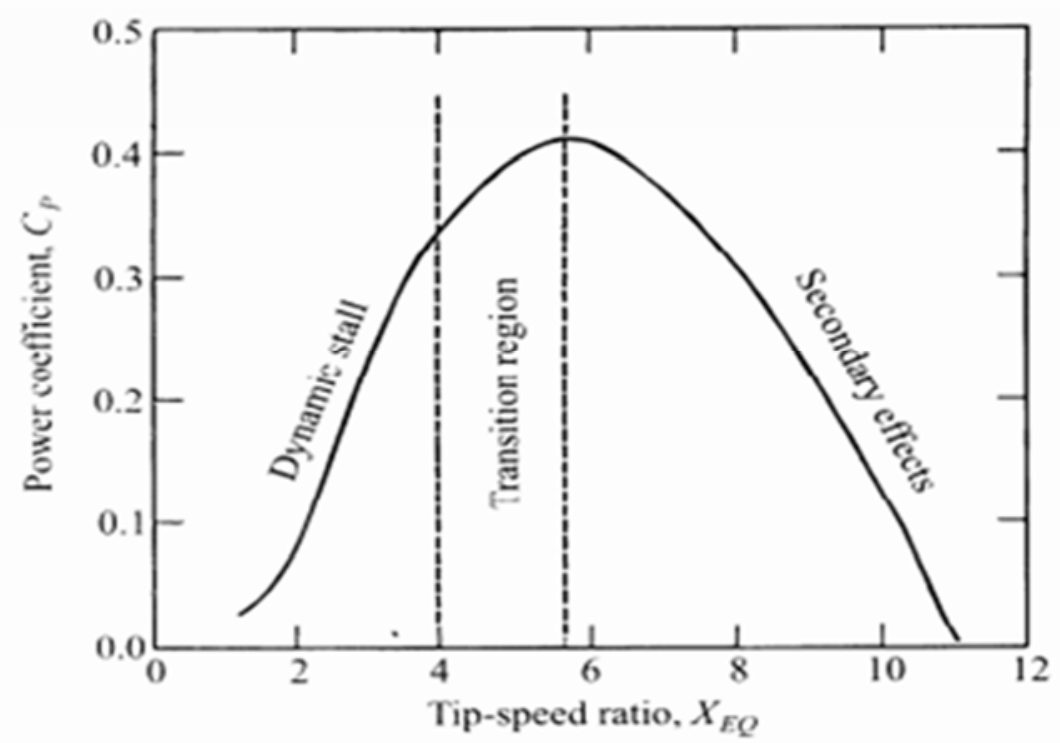

Figure 7. Coefficient de puissance en fonction du TSR pour une éolienne de type Darrieus, (PARASCHIVOIU, 2002).

\section{Conclusions}

Ce travail a porté sur les hydroliennes à axe verticale de type Darrieus avec des pales NACA4415. La géométrie utilisée dans ce travail a été réalisée en $2 \mathrm{D}$, elle a été faite avec deux domaines différents, un fixe (milieu de l'écoulement) et l'autre mobile (zone rotative 


\section{Thème 5 - Énergies et ressources marines}

de l'hydrolienne) pour pouvoir utiliser la technique de Sliding Mesh dans le solveur Fluent. Nous avons traité le cas du milieu infini puis le milieu fini.

Au niveau du modèle k- $\omega$ SST (dans ANSYS FLUENT, 2014), l'activation de la correction bas-Reynolds (LReC) nécessite un maillage raffiné avec $\mathrm{y}^{+}$de l'ordre de 1 . Nos simulations confirment que la non-activation de cette fonctionnalité conduit à un modèle haut-Reynolds avec utilisation d'une fonction de parois et impliquant un choix de $30<\mathrm{y}^{+}<300$. Dans ce cas, des valeurs faibles de $\mathrm{y}^{+}$entrainent des résultats incorrects. L'utilisation d'une fonction de paroi (modèle haut Reynolds) est possible dans le cadre des applications qui concernent l'écoulement loin des parois. Cependant, dans le cadre des machines tournantes (turbomachines en général et hydroliennes en particulier) où le calcul précis des forces (portances et trainées) est important, la résolution de la sous couche visqueuse est nécessaire (d'où un modèle bas-Reynolds).

En milieu infini (Profil NACA4415), nous avons vérifié le coefficient instantané sur un tour d'hydrolienne pour les 3 pales. Le résultat confirme celui de HAMMOUDI et al, (2016) sur l'ordre des pics du coefficient de moment instantané sur les pales.

En milieu fini, les résultats montrent que la correction avec un modèle bas-Reynolds ainsi qu'un choix d'une valeur de $\mathrm{y}^{+}=0,74$ a permis une amélioration du résultat qui réduit l'écart par rapport aux valeurs expérimentales de PATEL et al. (2017) jusqu'à la vitesse spécifique $\mathrm{TSR}=1.5$. Au-delà de cette valeur les $\mathrm{C}_{\mathrm{M}}$ numériques augmentent contrairement à celles obtenues expérimentalement avec des $\mathrm{C}_{M}$ qui continuent de décroitre jusqu'à une valeur presque nulle. Les simulations avec des TSR $>2$ ont été motivées par le fait que la puissance expérimentale maximale n'était que de $8 \%$ alors que dans la littérature les puissances obtenues étaient beaucoup plus importante. Ces simulations ont permis d'obtenir la courbe de puissance pour différentes valeurs de la vitesse spécifique ainsi que la puissance optimale $45 \%$ pour TSR=3. Cet écart semble être lié à une perte d'information au niveau du système expérimental pour des vitesses spécifiques plus importantes. Cependant pour confirmer ces résultats il faudrait continuer le travail par d'autres simulations et éventuellement des expérimentations en canal à houle/courants.

\section{Remerciements}

M. E. GHEDHAB tient à remercier l'ECAM-EPMI pour avoir financé cette étude dans le cadre de son stage de Master 2 Mécanique, option : Modélisation et simulation en mécanique des fluides et Transferts Thermiques, de l'UPEM - Université de Paris-Est Marne-la-Vallée. 


\section{XVİ̀mes Journées Nationales Génie Côtier - Génie Civil \\ Le Havre 2020}

\section{Références bibliographiques}

AIT MOHAMMED M. (2013). Etude des tuyères composites pour une conception optimale d'une hydrolienne à axe horizontal, Thèse de doctorat, Université de Bretagne Occidentale, 330 p. https://tel.archives-ouvertes.fr/tel-01493676

ANSYS FLUENT (2014). FLUENT_V16, User's Guide, Version 16.0. ANSYS, Inc. Canonsburg, PA, USA.

BELHACHE M., GUILLOU S., MOUAZE D., SANTA-CRUZ A., GRANGERERT P. (2012). Etude numérique de l'intégration d'hydroliennes (à axe vertical) en courant giratoire, XIIème Journées Nationales Génie Côtier - Génie Civil, Cherbourg, 2012, pp 819-826. https://doi.org/10.5150/jingcgc.2012.089-B

CARLIER C., MYCEK P., GAURIER B., GERMAIN G., PINON G., RIVOALEN E. (2014). Etude expérimentale et numérique du comportement d'hydroliennes à axe horizontal, XIIIème Journées Nationales Génie Côtier-Génie Civil, Dunkerque, 2014, pp 727-734. https://doi.org/10.5150/jngcgc. 2014.079

HAMMOUDI Z., ABSI R., EL ABBASSI I., DARCHERIF A. M. (2016). Simulation bidimensionnelle d'une hydrolienne à axe vertical dans un courant marin, XIVème Journées Nationales Génie Côtier - Génie Civil, Toulon, 2016, pp 487-496. https://doi.org/10.5150/jngcgc.2016.055

KHAN M. J., BHUYAN G., IQBAL M. T., QUAICOE J.E. (2009). Hydrokinetic energy conversion systems and assessment of horizontal and vertical axis turbines for river and tidal applications: A technology status review, Applied Energy, vol. 86 (10), pp 1823-1835. https://doi.org/10.1016/j.apenergy.2009.02.017

LAIN S., OSORIO C. (2010). Simulation and evaluation of straight-bladed Darrieustypes cross flow marine turbine, Journal of Scientific \& Industrial Research, vol. 69 (12), pp 906-912. http://nopr.niscair.res.in/handle/123456789/10657

MENCHACA ROA A. (2011). Analyse numérique des hydroliennes à axe vertical munies d'un carénage, Thèse de doctorat, Université de Grenoble, 217 p. https://tel.archivesouvertes.fr/tel-00685850

MENTER F.R. (1994). Two-equation eddy-viscosity turbulence models for engineering applications, AIAA Journal, vol. 32 (8), pp 1598-1605. htps://doi.org/10.2514/3.12149

NGUYEN V.T., GUILLOU S., SANTA CRUZ A. (2014). Simulation numérique d'un parc pilote d'hydroliennes avec des vitesses de courant extrêmes : application au cas du Raz Blanchard, XIIIème Journées Nationales Génie Côtier -Génie Civil, Dunkerque, 2014, pp 769-778. https://doi.org/10.5150/jngcgc.2014.084

PARASCHIVOIU I. (2002). Wind turbine design with emphasis on Darrieus concept, Polytechnic International Press, Montreal, 2002, 590 p.

PATEL V., ELDHO T., PRABHU S. (2017). Experimental investigations on Darrieus straight blade turbine for tidal current application and parametric optimization for hydro farm arrangement, International Journal of Marine Energy, vol. 17, pp 110-135, https://doi.org/10.1016/jijome.2017.01.007 
Thème 5 - Énergies et ressources marines

SHIONO M., SUZUKI K., KIHO S. (2000). An experimental study of the characteristics of a Darrieus turbine for tidal power generation, Electrical Engineering in Japan, Vol. 132(3), pp 38-47. https://doi.org/10.1002/1520-6416(200008)132:3<38::AID-EEJ6>3.0.CO;2-E 